\title{
Sarcoidosis with complications
}

\author{
Andreu Fernández-Codina • Carmen Aleman • \\ Macarena Simón-Talero • Maria Teresa Salcedo-Allende • \\ Iago Pinal-Fernández • Antonio Segura-García
}

Received: 27 January 2014/ Accepted: 20 February 2014/Published online: 12 March 2014

(C) SIMI 2014

A 40-year-old man of Pakistani origin presented with a 2-week history of fever $\left(39.5^{\circ} \mathrm{C}\right)$, malaise and arthralgias. He had been living in Spain for 16 years, and he had not travelled to a foreign country in the prior 4 years. Three days before, conjunctival erythema and multiple painful and violaceous-erythematous nodules (red arrows in Panel B) appeared in both pretibial regions, preventing him from walking because of pain. Physical examination showed bilateral metacarpophalangeal, wrist, knee and ankle arthritis. Ophthalmologic examination showed bilateral anterior scleritis, with preserved visual acuity and without signs of uveitis (Panel A). A chest X-ray study demonstrated bilateral hilar and mediastinal adenopathies (blue arrows in Panel C). He was admitted to the internal medicine ward. Serum angiotensin conversor enzyme levels were normal. Human immunodeficiency virus serologies, Mantoux test, three sputum cultures with Ziehl-Neelsen

Electronic supplementary material The online version of this article (doi:10.1007/s11739-014-1067-6) contains supplementary material, which is available to authorized users.

A. Fernández-Codina $(\bowtie) \cdot$ C. Aleman · M. Simón-Talero · I. Pinal-Fernández · A. Segura-García

Servicio de Medicina Interna, Unidad de Enfermedades

Autoinmunes Sistémicas, 3a planta edificio Hospital General,

Hospital Universitari Vall d'Hebron, Universitat Autònoma de

Barcelona, Passeig de la Vall d'Hebron 119-129,

08035 Barcelona, Spain

e-mail: andreu.fernandez@vhebron.net

A. Fernández-Codina $\cdot$ M. Simón-Talero · I. Pinal-Fernández Emergency Department, Hospital Universitari Vall d'Hebron, Universitat Autònoma de Barcelona, Barcelona, Spain

M. T. Salcedo-Allende

Pathology Department, Hospital Universitari Vall d'Hebron,

Universitat Autònoma de Barcelona, Barcelona, Spain staining and a bronchoalveolar lavage (BAL) ruled out infectious disease. The CD4/CD8 ratio in the BAL was 2:1. A transbronchial biopsy of an adenopathy showed nonnecrotizing granulomas, without microbiological findings. The patient was diagnosed as a case of sarcoidosis, with the clinical presentation of a Löfgren's syndrome. Lung alterations corresponded to a stage II pulmonary sarcoidosis. The patient was treated with dexamethasone eye drops and oral dexketoprofen. Scleritis, temperature, joint swelling and arthralgias disappeared, and he was discharged home. Two weeks later, he again developed fever and mild dyspnoea. Lung functional tests were normal, with the exception of a slightly lowered carbon monoxide lung diffusion (71\%), which was normalized after volume correction. After ruling out etiologies different from sarcoidosis, oral prednisone $20 \mathrm{mg} /$ day was administered for a 4-week course as indicated in refractory disease, with total disappearance of symptomology. Adenopathies and parenchymal lung alterations remained stable. Currently, he has completed the treatment, and remains asymptomatic.

We have presented a case of sarcoidosis with a typical Löfgren's syndrome, consisting of hilar adenopathies, erythema nodosum and polyarthralgia or polyarthritis. This syndrome is found more frequently in women, and the course is usually benign often with [1] spontaneous remission. Anterior scleritis is an unusual ophthalmologic finding in sarcoidosis, and it has been related with Löfgren's syndrome in a few cases [2]. Sporadic case reports [3] describe a good response to corticosteroids, but in this case, the scleritis responded well to non-steroidal antiinflamatory medication. The treatment of sarcoidosis with corticosteroids is not recommended [4] for stage II pulmonary sarcoidosis because $50 \%$ of the untreated patients heal spontaneously. Nevertheless, symptomatic 

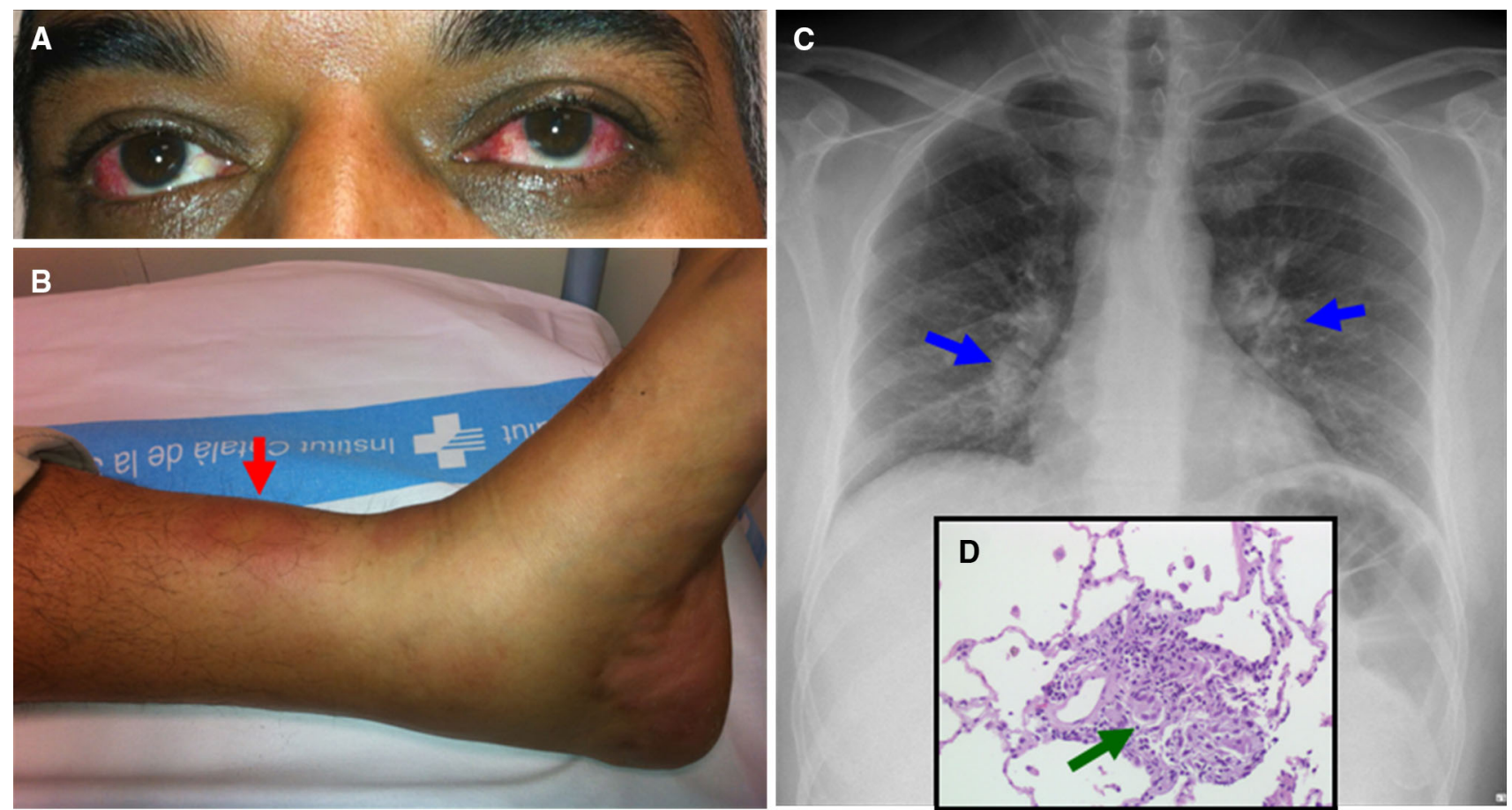

stage III, all stage IV pulmonary sarcoidosis, and patients

\section{References}

with lower grades of pulmonary sarcoidosis but with severe extrapulmonary sarcoidosis or with incapacitating disease, should be treated with $20-40 \mathrm{mg}$ /day of oral prednisone for a month. A 3-6 months slow prednisone tapering down is started thereafter. Unfortunately, some patients may still have relapses, and develop steroiddependent sarcoidosis.

Conflict of interest The authors state that they have no conflicts of interest and that they have not received any funding from others. The patient signed a permission form, authorising the scientific use of his pictures.

1. Mañá J, Gómez-Vaquero C, Montero A et al (1999) Löfgren's syndrome revisited: a study of 186 patients. Am J Med 107:240

2. James DG, Neville E, Langley DA (1976) Ocular sarcoidosis. Trans Ophthalmol Soc UK 96:133-139

3. Dursun D, Akova YA, Bilezikçi B (2004) Scleritis associated with sarcoidosis. Ocul Immunol Inflamm 12:143-148

4. Bradley B, Branley HM, Egan JJ et al (2008) Interstitial lung disease guideline: the british thoracic society in collaboration with the thoracic society of Australia and New Zealand and the Irish thoracic society. Thorax 63(Suppl 5):v1 